\title{
Feasibility of measuring Event Related Desynchronization with Electroencephalography during Walking
}

\author{
M. Severens, B. Nienhuis, P. Desain and J. Duysens
}

\begin{abstract}
Brain Computer Interfaces could be useful in rehabilitation of movement, perhaps also for gait. Until recently, research on movement related brain signals has not included measuring electroencephalography (EEG) during walking, because of the potential artifacts. We investigated if it is possible to measure the event Related Desynchronization (ERD) and event related spectral perturbations (ERSP) during walking. Six subjects walked on a treadmill with a slow speed, while EEG, electromyography (EMG) of the neck muscles and step cycle were measured. A Canonical Correlation Analysis (CCA) was used to remove EMG artifacts from the EEG signals. It was shown that this method correctly deleted EMG components. A strong ERD in the mu band and a somewhat less strong ERD in the beta band were found during walking compared to a baseline period. Furthermore, lateralized ERSPs were found, depending on the phase in the step cycle. It is concluded that this is a promising method to use in BCI research on walking. These results therefore pave the way for using brain signals related to walking in a BCI context.
\end{abstract}

\section{INTRODUCTION}

In rehabilitaiton medicine, restoring gait functions is very important, because this determines if patients can become independent. Recently the idea has arisen that Brain Computer Interfaces could be used for the rehabilitation of gait. Walking is a very automated process and it is argued that it is controlled at a subcortical level [see 1 for a review]. Animal studies in decerebrated cats suggest that central pattern generators in the spinal cord are involved. Similar results have been found in humans. In complete spinal cord patients, walking movements can be induced by stimulating the spinal cord or by putting them on a treadmill [2, for a review see 1]. This suggests that walking is at least partly controlled at subcortical levels. So the question is whether it is possible to measure brain activations during walking.

* This research is supported by the BrainGain Smart Mix Programme of the Netherlands Ministry of Economic Affairs and the Netherlands Ministry of Education, Culture and Science.

M. Severens is with the Research Development \& Education department, Sint Maartenskliniek, Nijmegen, The Netherlands and the Donders Centre for Cognition, Radboud University Nijmegen, The Netherlands (phone: +31 24 3659329; fax: +31 24 3659154; e-mail: m.severens@maartesnkliniek.nl).

B. Nienhuis, is with the Research Development \& Education department, Sint Maartenskliniek, Nijmegen, The Netherlands (e-mail: b.nienhuis@maartensklineik.nl).

P. Desain is with the the Donders Centre for Cognition, Radboud University Nijmegen, The Netherlands (e-mail: p.desain@donders.ru.nl).

J. Duysens is with the Department of Kinesiology, KU Leuven, Belgium and with the Research Development \& Education department, Sint Maartenskliniek, Nijmegen (email: jacques.duysens@faber.kuleuven.be)
Several previous studies have shown that there are activations in cortical areas involved in walking using functional magnetic resonance imaging (fMRI) [3] and near infrared spectroscopy (NIRS) [4,5]. However, the temporal resolution of these hemodynamic methods is in the order of seconds. To be able to see changes within a step cycle a method with a higher temporal resolution, such as electroencephalography (EEG), is necessary. The difficulty with EEG measurements during walking is that EEG is susceptible to contamination with artifacts from facial and neck muscles and movement artifacts from the electrodes and cables. Recently some attempts have been made to overcome these problems and brain signals related to walking have been reported. Kinematics from lower limb joints could be inferred from EEG signals [6]. Furthermore, gait event related spectral perturbations (ERSP) in mu, beta and gamma band were found that are coupled to the gait cycle $[7,8]$. Independent component analysis (ICA) and dipole analysis were used to ensure that the signals were not contaminated with artifacts.

The most appropriate candidate for the brain signal that could be used in BCI in rehabilitation is the event related desynchronization (ERD). This ERD is found in the $\mathrm{mu}$ and beta frequency range during movement and imagination of movement $[9,10]$. These ERD signals have not yet been investigated in walking. In this study we will therefore investigate the possibility to measure the ERD during walking. Furthermore the ERSPs will be investigated with methods that can easily be applied in a BCI setting. Canonical correlation analysis (CCA), a blind source separation method which provides an estimate of the autocorrelation in a signal [11], will be used to separate electromyography (EMG) and EEG sources.

\section{MEthoDS}

\section{A. Subjects and Design}

Six healthy subjects aged between 20-26 years (mean 21.6, SD 2.3), with no history of neurological disease or lower limb pathology participated in this experiment. All subjects gave written informed consent before the start of the experiment.

Since patients usually walk slowly, we instructed the subjects to perform walking at a slow speed on a treadmill (ENRAF Nonius, Type EN-tred Reha), with a step frequency of $1.4 \mathrm{~Hz}$. The speed of the treadmill for the walking condition was adjusted to a comfortable speed for each subject before the start of the experiment. The walking speed was on average $2.8(+/-0.2) \mathrm{km} / \mathrm{h}$. Five trials of approximately two minutes of walking were performed. Each trial consisted of a baseline period in 
which the subjects stood still and looked at a fixation cross on a computer screen. After 9 seconds an instruction appeared on the screen for 1 second. Then again a fixation cross was shown on the screen indicating that the subjects had to start the task. The treadmill started, which took about 7 seconds to come to speed. Some 120 seconds after starting the treadmill it was slowed down and stopped again. Additionally a metronome was used for about 15 seconds as soon as the second fixation cross was shown, to help the subjects synchronize to the required step frequency.

\section{B. Methods}

EEG was recorded with 62 electrodes with the ground placed on the AFz-electrode position. The EEG signals were amplified using a TMSi Refa-72 amplifier (Twente Medical Systems International, The Netherlands) and digitized at a sampling rate of $500 \mathrm{~Hz}$, referenced to an average of all channels, with a low-pass filter at $135 \mathrm{~Hz}$. The electromyography (EMG) of the neck muscles was recorded with ZeroWire wireless bipolar electrodes (Aurion SRL, Italy). It was sampled at $2 \mathrm{KHz}$, filtered between $10-1000 \mathrm{~Hz}$, and then streamed to the EEG amplifier, where it was resampled at $500 \mathrm{~Hz}$. For gait phase detection, wireless foot contact sensors were used (Footswitch, Zerowire, Aurion SRL, Italy) which were attached at the level of the calcaneus and between the dorsal heads of metatarsal I and II on both feet.

\section{Analyses}

Analyses were performed using the Fieldtrip toolbox for EEG/MEG-analysis [12] and Matlab (the MathWorks Inc.). The EEG and EMG data were analysed in a trial and step cycle epoch. First, whole trials were analysed with baseline (standing quietly, +/- 9 seconds) and walking period $(+/-120$ seconds). Second, trials were cut into smaller epochs based on the footswitch data, starting with

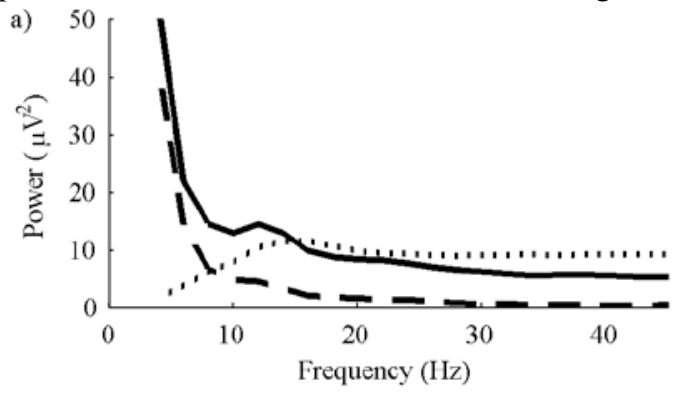

c)

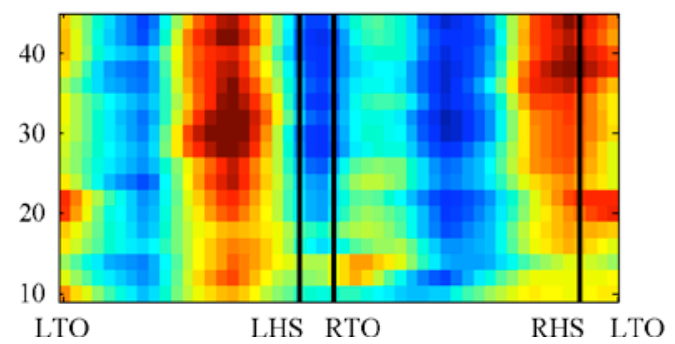

a left toe off (LTO), until the next left toe off (comparable to [7]). Furthermore, several other phases in the step cycle were detected, such as left heel strike (LHS), right toe off (RTO) and right heel strike (RHS). If a clear step cycle phase could not be detected from the footswitch data, the epoch was discarded from further analysis. Noisy EEG trials were removed by visual inspection of the variance in each trial and epoch.

During walking the neck muscles are active and they can contaminate the EEG signal. We used a CCA to detect EMG components [11]. This method is based on the assumption that EMG signals have low autocorrelation, while EEG signals have higher autocorrelation. First, a CCA decomposition is performed on the EEG data, resulting in a set of components that is ordered based on the autocorrelation of each component. To select the EMG components, the spectral power in the EEG frequency band $(0.5-15)$ is compared with the EMG frequency band (15-30) for each component. When the power in the EMG is 4 times stronger than the power in the EEG band, the component was marked as an EMG component and removed. Subsequently, the EEG data was reconstructed into a "channel by time" representation. The same EMG components were removed for both the trial and step cycle analysis. On the cleaned EEG data a line noise band stop filter $(50 \mathrm{~Hz})$ was applied and linear trends were removed. For each trial and epoch, a baseline correction was applied by subtracting the mean potential over time to remove offsets. For the trial analysis the spectrogram was calculated with a convolution method using a sliding Hanning window of $1 \mathrm{~s}$, with an interval of $0.2 \mathrm{~s}$. This spectrogram was calculated per trial and then averaged. For the step cycle epochs, spectrograms were calculated per step cycle, with a sliding window and interval of 0.5 and $0.1 \mathrm{~s}$ respectively. These were then stretched using linear interpolation to a standard step cycle period and then averaged over trials.
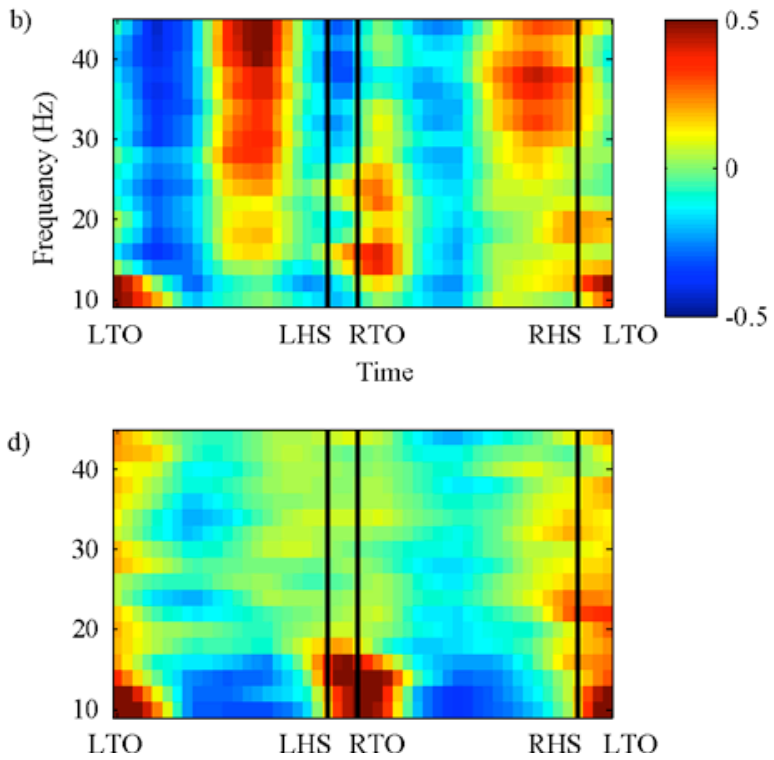

Figure 1. Spectra of an occipital channel (O1) before and after CCA component rejection and EMG spectra for a typical subject. (a) Power spectra of the EEG in the O1 electrode before (solid line) and after (dashed line) CCA component rejection are shown along with the EMG spectra in the left neck muscle (dotted line). Time frequency representations of the (b) EMG, (c) O1 elctrode before, and (d) after CCA component rejection. Colors indicate the relative change in the power. Blue colors indicate desynchronizations, red colors indicate synchronizations. 


\section{RESULTS}

Because neck muscle activity during walking can contaminate the EEG signal, it is important that this EMG activity is correctly removed from the EEG signal. A property of EMG signals is that they have a broad spectrum and white noise properties [13]. In contrast, the power of EEG signals typically is inversely related to the frequency. This allows us to determine if the CCA component rejection method correctly removed the EMG components from the EEG data. Fig. 1 shows spectral data for a typical subject. Before rejection the EEG data in occipital channels contained strong power in frequencies in the EMG range, similar to the EMG spectrum (Fig. 1a). After CCA component rejection, the power in these frequencies was reduced. This can also be seen in the time frequency representations of the step cycles (Fig. 1b-d). Before rejection, strong broad-spectrum bands of synchronization and desynchronization were found in both the occipital channel and the EMG channel. After rejection, these bands were greatly reduced in the occipital channel. Now desynchronizations and synchronizations are revealed in the $10-18 \mathrm{~Hz}$ band.

In the trial analysis a clear desynchronization in the mu band during the movement could be seen, compared to the baseline period (see Fig. 2a). Furthermore a desynchronization in the beta band was also seen, although less clear. The topography of the beta and mu ERD was slightly different (see Fig. $2 \mathrm{~b}$ and $\mathrm{c}$ respectively). The beta ERD was strongest above the lateral motor cortex (C3 and $\mathrm{C} 4)$. The mu ERD was strongest above the central motor cortex $(\mathrm{Cz})$.

During gait the ERSPs were found in the step cycle above the motor cortex (see Fig. 3a-b). In the swing phase (between toe off and heel strike) a desynchronization occurred in the mu and beta band. Just before heel strike and in the double support phase an increase in the power in lateralized depending on the phase in the step cycle (see Fig. 3c). Desynchronization was strongest in electrodes above the motor cortex in the swing phase of the contralateral leg (eg. the desynchronization between RTO and RHS is strongest around C3). Just before heel strike and in the double support phase, the increments in activity

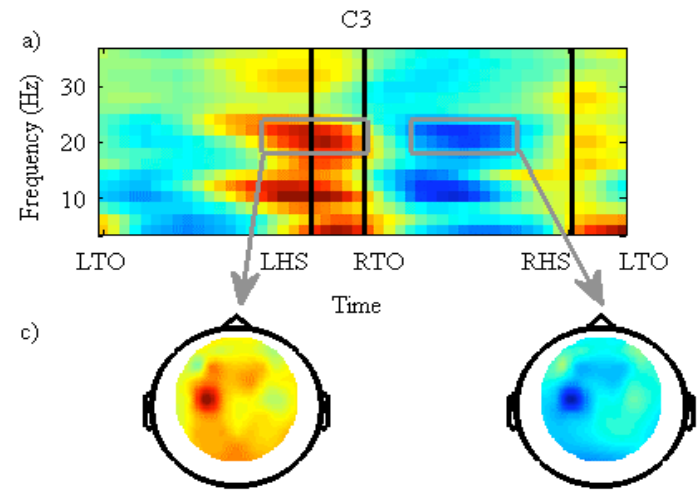

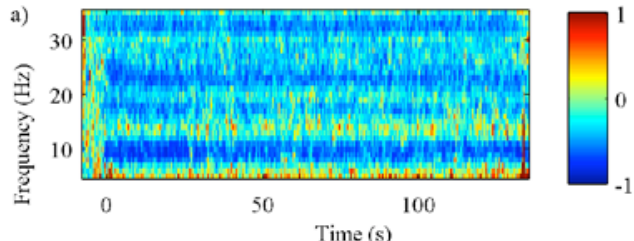

b)



c)

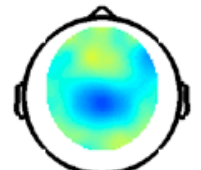

Figure 2. (a) Power spectrum and topographies in the (b) beta (18-26 $\mathrm{Hz})$ and (c) mu (8-12 Hz) band for the trial analysis averaged over all subjects. In (a) the time before zero was the baseline period. At zero seconds, the walking task started. Blue colors indicate desynchronization, red colors indicate synchronization.

were strongest in the hemisphere ipsilateral to the heel strike (eg. the beta activity just before and after RHS was strongest around $\mathrm{C} 4)$.

The ERSPs are quite similar in electrodes directly above the motor cortex before and after CCA. However, before CCA the topography of the beta activity was contaminated with artifacts in frontal and occipital electrodes, obscuring the effect above the motor cortex (see Fig 4). In the topography obtained after CCA, the muscle artifacts were removed.

\section{DISCUSSION}

We investigated the possibility of measuring ERD and ERSPs during slow walking. An ERD in the mu and beta band was found, although the latter one was less clear. Furthermore ERSPs were found in the step cycle.

The differences in ERD between mu and beta band could be caused by inter-individual differences. For the beta band, the peak frequency of the beta band differed among subjects. Some even showed synchronization in the frequencies where others showed desynchronization. Averaging over subjects could therefore have led to a less clear ERD in the beta band. This inter-individual difference in the frequencies of the ERD has been reported before in healthy subjects [14] and paraplegic patients [15]. In the current study, the peak frequencies of the ERD in the mu band were more similar amongst subjects.
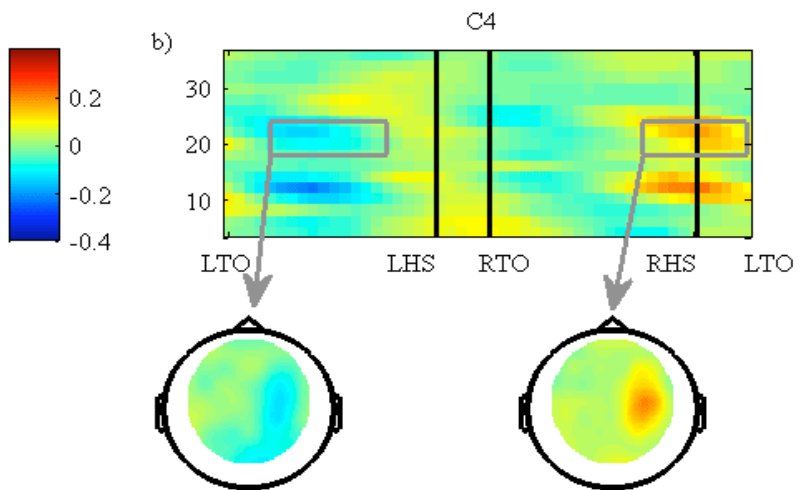

Figure 3. Grand average ERSP over all subjects during the step cycle for the (a) C3 and (b) C4 electrode and (c) topographies of the beta (18-24 Hz) activity for the different phases in the step cycle. Black lines indicate the different phases in the step cycle. Squares indicate the time and frequency selection for each topography. Blue colors indicate desynchronization, red colors indicate synchronization. 
a)
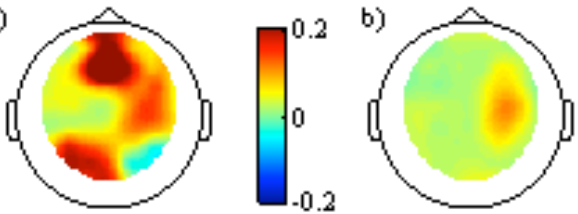

Figure 4. Differences in topographies a) before, and b) after CCA component rejection. As an example the beta (18-24 Hz) activity around the right heel strike is shown (see also Fig 3c). Blue colors indicate desynchronization, red colors indicate synchronization.

A second interesting finding regarding the ERD is that we found a different spatial distribution in the mu and beta band. Whether this difference is caused by the interindividual differences, or whether it represents a true difference in the generation of the mu and beta ERD, remains to be investigated.

In correspondence with [8] we found ERSPs in mu and beta band that were coupled to the gait cycle. Furthermore we could detect differences between left and right swing and double support phase, without using ICA and source analysis techniques. These changes over time give information about the frequency and phase of walking and therefore, could be useful in a BCI for rehabilitation of gait.

The CCA seems a promising method to remove EMG components from the EEG data during walking. Although former studies $[7,8]$ already showed that the brain signals during walking can be investigated while the influence of EMG artifacts is minimized, the methods are not easily usable in an online BCI. First, ICA most of the time needs a large amount of data to estimate the components, and the optimization makes it computationally heavy and timeconsuming. Second, dipole fitting is needed to select relevant components and reject the ones that have sources outside the brain, which is also time-consuming. The CCA method that we used, on the other hand, could in principle be used on single trials, as [11] have shown when measuring EEG during speech production. This could be an advantage in BCI.

Although great care was taken to exclude muscle and movement artifacts, it is still possible that some artifacts remain in the EEG data. The modulations in the lower frequency band in the occipital channel that were revealed after CCA are probably still due to remaining artifacts. These modulations could still come from muscle activity, or be caused by movement artifacts of the electrodes and head. It is unlikely that these modulations are related to brain activity involved in walking, because they are seen in the occipital electrodes. Unfortunately we did not record movements of the head. At slow speeds of walking, these movements are probably not very important. However, in future research, especially for higher speeds of walking and, it would be advisable to measure these movements in order to remove the artifacts from the EEG signals by decorrelation or another artifact removal method.

\section{CONCLUSION}

It is shown that it is possible to detect ERD during slow walking movements. The lateralization in the gait ERSPs that we found could be useful for brain computer interfaces, to detect and give feedback about the phase of the gait cycle. It remains to be seen if these signals can also be detected when imagining walking or in patients with paresis of the legs. A next step would be to investigate if these signals are strong enough to be detected in only a couple of trials.

\section{ACKNOWLEDGMENT}

We thank Erwin Peters for help with the measurements and Jason Farquhar for helpful tips on data analysis.

\section{REFERENCES}

[1] J. Duysens, and H.W. Van de Crommert, "Neural control of locomotion; The central pattern generator from cats to humans," Gait Posture, vol. 7(2), pp. 131-141, 1998.

[2] V. Dietz, G. Colombo, L. Jensen, and L. Baumgartner, "Locomotor capacity of spinal cord in paraplegic patients," Annals of Neurology, vol. 37, pp. 574-582, 1995.

[3] M. Bakker, F.P. De Lange, R.C. Helmich, R. Scheeringa, B.R. Bloem, and I. Toni, "Cerebral correlates of motor imagery of normal and precision gait," Neuroimage, vol. 41(3), pp. 998-1010, 2008.

[4] I. Miyai, H.C. Tanabe, I. Sase, H. Eda, I. Oda, I. Konishi, Y. Tsunazawa, T. Suzuki, T. Yanagida, and K. Kubota, "Cortical mapping of gait in humans: a near-infrared spectroscopic topography study," Neroimage, vol. 14(5), pp. 1186-1192, 2001.

[5] M. Suzuki, I. Miyai, T. Ono, I. Konishi, T. Kochiyama, and K. Kubota, "Prefrontal and premotor cortices are involved in adapting walking and running speed $\mathrm{n}$ the treadmill: and optical imaging study," Neuroimage, vol. 23(3), pp. 1020-1026, 2004.

[6] A. Presacco, R. Goodman, L. Forrester, and J.L. Contreras-Vidal, "Neural decoding of treadmill walking from noninvasive electroencephalographic signals," J Neurophysiol, vol. 106, pp. $1875-1887,2011$

[7] J.T. Gwin, K. Grasman, S. Makeig, and D.P. Ferris, "Removal of movement artifact from high-density EEG recorded during walking and running," J Neurophysiol, vol. 103, pp. 3526-3534, 2010.

[8] J.T. Gwin, K. Grasman, S. Makeig, and D.P. Ferris, "Electrocortical activity is coupled to gait cycle phase during treadmill walking," Neuroimage, vol. 54, pp. 1289-1296, 2011.

[9] G. Pfurtscheller, and C. Neuper, "Event-related synchronization of mu rhythm in the EEG over the cortical hand area in man," Neurosci Lett, vol.174(1), pp. 93-96, 1994.

[10] G. Pfurtscheller, C. Brunner, A. Schlögl, and F.H. Lopes da Silva, "Mu rhythm (de)synchronization and EEG single-trial classification of different motor imagery tasks," Neuroimage, vol. 31(1), pp. 153-159, 2006.

[11] M. De Vos, s. Riès, K. Vanderperren, B. Vanrumste, F.X. Alario, S. Van Huffel, and B. Burle, "Removal of muscle artifacts from EEG recordings of spoken language production," Neuroinfromatics, vol. 8(2), pp. 135-150, 2010.

[12] R. Oostenveld, P. Fries, E. Maris, and J.M. Schoffelen, "FieldTrip: Open source software for advanced analysis of MEG, EEG and invasive electrophysiological data," Computational Intelligence and Neuroscience, 2011.

[13] I.I. Goncharova, D.J. McFarland, T.M. Vaughan, and J.R. Wolpaw, "EMG contamination of EEG: spectral and topgraphical characteristics," Clin Neurophysiol, vol. 114, pp. 1580-1593, 2003.

[14] C. Neuper, and G. Pfurtscheller, "Evidence for distinct beta resonance frequencies in human EEG related to specific sensorimotor cortical areas," Clin Neurophysiol, vol. 112, pp. 2084-2097, 2001.

[15] G.R. Müller-Putz, D. Zimmermann, B. Graimann, K. Nestinger, G. Korisek, and G. Pfurtscheller, "Event-related beta EEG-changes during passive and attempted foot movements in paraplegic patients", Brain Research, vol. 1137, pp. 84-91, 2007. 